\title{
FINAL REMARKS ON THE BINARY HYPOTHESIS \\ FOR THE BE STARS
}

\author{
MIROSLAV PLAVEC \\ Dept. of Astronomy, University of California, Los Angeles, Calif., U.S.A.
}

\begin{abstract}
The hypothesis that a significant fraction of Be stars are interacting binaries is discussed in general terms. The author believes that the observed Be stars are a mixture of three different types of objects. The weakest point of the single-star hypothesis is probably the difficulty in explaining the quasi-periodic $V / R$ variations; the weakest point of the binary hypothesis appears to be the statistical expectancy of a fairly high number of eclipsing systems among them.
\end{abstract}

From various comments made during this Symposium, I gather that the binary model for Be stars is beginning to be taken seriously. This enables me to change somewhat this closing talk of the session. I feel that it is no longer necessary to call attention repeatedly to the binary model; instead, I may try to be more impartial. In other words, I will not follow the radicalism of Harmanec and Kř́ž who propose the working hypothesis that all $\mathrm{Be}$ stars are binaries. Rather, I will adhere to the suggestion made several times in the past (Plavec and Horn, 1969; Kř́iž, 1969; Plavec, 1970a; Plavec et al., 1973) that a significant number of Be stars are probably interacting binaries. This formulation admits the existence of several possible models, so that we would have objects similar in appearance but different in origin. This is not too satisfactory at first sight, but after all, a Be star is simply a B star surrounded by an extended envelope. Why should we insist that the envelope can be formed in only one way? In fact, I can think of three possible ways: a rapidly rotating single star; an interacting binary system; and a young star still surrounded by the remnants of its original cocoon (i.e., Herbig's Ae and Be stars).

The idea of the binary model was conceived in 1968-69 not because the classical model proposed by Struve was deemed unacceptable. Rather, theoretical results of modeling of interacting binaries indicated that here we have a process which could also lead to something similar to a $\mathrm{Be}$ star. The results of evolutionary model calculations for interacting binaries have been reviewed several times (see, e.g., Plavẹc, 1970b, or Paczyński, 1971), so that I can be quite brief. The more massive star of a binary system is the first component to evolve away from the near vicinity of the zero-age main sequence; as it evolves, it expands until it fills its critical Roche lobe. If the system is very close, the orbital period being less than one day, the more massive component is trapped while it is still a main-sequence star. It begins to lose mass on a thermal time scale. The other component cannot be much smaller, being a main-sequence star in a system where the separation of the components is only of the order of 10 solar radii or less. The gas streaming out of the more massive star near its first Lagrangian point is deflected owing to the rotation of the system, and generally impinges on the less massive star under an oblique angle. It will probably accelerate that star's axial rotation, but an extensive circumstellar envelope cannot be expected in this case. Accretion causes the accreting star to swell and very soon it will fill its critical lobe, too (Benson, 1970), and we will have a contact system. 
Much more interesting for us is the case when the binary system is wider to begin with, having an orbital period of at least several days. In this case the more massive component will not reach the Roche limit until it is burning hydrogen in a shell surrounding a helium-rich core. The system is generally wider, the separation of the components being between 10 and 100 solar radii, and this separation grows in the later stages of mass transfer, when the mass-losing star already becomes the less massive component. It is these stages which interest us in connection with the $\mathrm{Be}$ phenomenon. For now the material leaving it comes into the vicinity of the mass-accreting star with excess angular momentum, and therefore tends to form a disk around that star. The disk may just about fill the critical Roche lobe of the accreting star, but is relatively flat, the material being concentrated near the orbital plane. Our idea is that this disk will produce emission and/or shell absorption lines typical for Be stars and shell stars.

That this mechanism works is indicated by the $\mathrm{H} \alpha$ profiles shown in the introductory lecture (p. 1). There is every reason to believe that RZ Sct, V 367 Cyg, W Ser, AU Mon, and RX Gem are interacting binaries of roughly the type described above. Observed profiles like these can now be fairly easily obtained thanks to modern image tubes; one generation ago, $\mathrm{H} \alpha$ was much more difficult to observe in fainter stars. Nevertheless, Struve, Merrill, McLaughlin and others did occasionally comment on the similarity between the spectral features in certain Be stars or shell stars and in certain eclipsing binaries. Why, then, did no one suggest a binary model for $\mathrm{Be}$ stars already thirty years ago? I think the reason is the observed rapid rotation of the Be stars. Rapid rotation is an inherent property of many B and A stars. We do not have to postulate a binary model to explain rapid rotation. In fact, close binary stars are often associated with slow rotation, since the tidal force tends to synchronize the axial rotation with orbital revolution. I think that the rapid rotation still represents the strongest argument in favor of Struve's classical model for many Be stars. It is only necessary to find an additional weak force which would push the material into the envelope. Various possibilities were investigated by Limber and Marlborough (1968). In recent years, stellar winds became very popular. However, in agreement with Dr Conti's comments, I must ask how the stellar wind can explain the cyclic changes observed in some of the envelopes, particularly in shell stars. We observe the material to drift slowly outwards, but then the motion stops and may even be reversed, as in 48 Librae. Also, while the parental star appears to be the same, a new shell may begin to form after years of inactivity, as in Pleione or very recently in $o$ And. These phenomena are hardly consistent with a uniformly blowing stellar wind.

One may object that model calculations for mass-transferring binaries do not predict this type of mass transfer rate variations either. However, one should bear in mind that the computing codes used cannot describe changes occurring on a time scale substantially shorter than the thermal time scale of the stars. In an approach where effects on the dynamical time scale were included, Bath $(1969,1972)$ repeatedly obtained very significant instabilities, and the observed period fluctuations in many Algol binaries indicate that short-term variations in the transfer rate are likely (Biermann and Hall, 1973). 
Without going into details, one can, I think, easily see that the binary model for the Be stars is more promising than the single-star model for objects displaying periodic or quasi-periodic changes such as the $V / R$ variations. In a rapidly rotating single star, one has just one fundamental period, namely the period of rotation of the star; and it is difficult to understand why the envelope should not be symmetric with respect to the equatorial plane and to the axis of rotation. This is why it was necessary to introduce some asymmetry by postulating an elliptical ring. A simple geometrical model of such a ring does indeed explain the $V / R$ variation at least qualitatively or semi-quantitatively, but nobody has explained yet how such a ring can exist as a three-dimensional feature, how it can be formed and how it can persist for a sufficiently long time. In the solar system, we have analogical structures in the meteor streams. However, there it is easy to understand why the ring is relatively narrow, for it has been formed by gradual or at most mildly violent disintegration of a comet moving in an elliptical orbit. Even so, these meteor streams show fairly large scatter of orbits at the aphelion, where the spatial density of the particles is much smaller than near the perihelion where the stream moves faster but is much more concentrated.

Harmanec and Kłriž have shown that a structure somewhat similar to a ring may be more easily formed by a certain type of mass influx from a mass-losing component in an interacting binary. I hope to see soon a more detailed study showing that indeed this model, in a three-dimensional picture, does give not only the required profile shape and variation, but the required emission intensity as well. However, I believe that even the quasi-stationary state of a fully developed disk offers possibilities for periodic or cyclic spectral changes. We have now at least two different fundamental periods in the system, rotation and revolution. As the components revolve about the center of gravity, the disk is observed from different directions. No such symmetry as in the single-star model can exist, for the gravitational field is not symmetrical with respect to the central star of the disk. Moreover, we can anticipate streams in the system; the main stream transferring material between the components, a possible stream which may return part of the material back to the parental star, and another possible stream (or streams?) which may carry part of the gas away from the system (see Figure 8 on page 9).

Naturally, very much must be done to convert this crude picture into something that can be compared with observations. This field is far from inactive, but hydrodynamics of gas streaming in close binaries is a very difficult problem. Moreover, theorists working in this field are much more eager to investigate these processes in $\mathrm{X}$-ray binaries, and ordinary binary systems have been rather neglected. An excellent discussion of the problem, together with references to earlier work, can be found in a recent article by Lubow and Shu (1975). It is not yet clear how fast the disk dissipates if the influx of material ceases. In the picture offered by Lubow and Shu, the viscosity in the disk is small, and the disk dissipates slowly. Some students of $\mathrm{X}$-ray binaries, however, postulate very high viscosity which rapidly transfers angular momentum through the disk outwards, and dissipates the disk fast.

Even less well understood is the actual process of accretion of the mass from the disk by the star inside it. At the advanced stages of mass transfer, when the accreting 
star is small compared to the dimensions of the system, the accretion occurs from essentially circular Keplerian orbits at small relative speeds, and will not affect the axial spinning of the accreting star seriously. However, at the earlier stages the stream impinges on the accreting star directly and under an oblique angle, and may accelerate the star's, rotation significantly (Plavec, 1970a; Van den Heuvel, 1970). We have a good example of this process in the Algol-type eclipsing binary star $\mathbf{U}$ Cephei. This system consists of a mass-losing subgiant, spectral class about G8 IIIIV, and of a B7V component which accretes mass, and was studied in great detail by Batten (1974). The synchronized equatorial velocity of axial rotation of the B star would be about $60 \mathrm{~km} \mathrm{~s}^{-1}$; however, the great width of its photospheric lines indicates a velocity of rotation of about $310 \mathrm{~km} \mathrm{~s}^{-1}$. An almost identical velocity is indicated by the emission lines of the disk which surrounds the star. We have no model of the accretion process or of the structure of the star. We cannot say if this high speed of rotation is the property of only a thin surface layer - but the same uncertainty exists in the single-star model of Be stars.

But can U Cephei be called a bona-fide Be star? I think it can just now, because it recently developed strong emission lines (Batten et al., 1975; Plavec and Polidan, 1975) which are at times visible even outside of eclipse. If the system were observed at an angle substantially different from $90^{\circ}$, we would speak of a Be star in a spectroscopic binary system. The other star would probably be invisible except in the infrared. For a truly conspicuous Be star, the emitting region would have to be larger compared to the central star. This can be achieved in a binary system of larger size, therefore of longer period, at least $\mathbf{3 0}$ days or more. Two systems of this type are known for sure, namely AX Mon and 17 Lep. Their spectra are rather complex, however, and they are not typical Be stars. Part of this anomaly may be due to the large mass and very large size of the mass-losing component: in both cases this is a large red giant, fairly easily detectable in the red region of the combined spectrum.

If $\mathrm{AX}$ Mon or 17 Lep were to represent typical examples of binary Be stars, we would have a serious problem with the absence of eclipses in Be stars. Suppose we have a large number of identical binary systems with a random orientation of their orbital planes. Let $R_{1}$ be the radius of the mass-accreting star, $R_{2}$ the radius of the mass-losing star, and $\boldsymbol{A}$ their separation. Typically in the interacting binaries we are considering here, the mass-losing star will be a giant of a later spectral type, and therefore considerably larger in size than the accreting B star. The fraction of systems that will display at least partial eclipses is

$$
f_{p}=\left(R_{1}+R_{2}\right) / A,
$$

while for the probability of total eclipses we have similarly

$$
f_{t}=\left(R_{2}-R_{1}\right) / A \text {. }
$$

Grazing partial eclipses will be invisible and $R_{1} \ll R_{2}$, so that we can estimate the fraction of eclipsing systems to be roughly

$$
f_{e}=R_{2} / A
$$

But the cool component is assumed to fill its critical Roche lobe, so that its fractional 
radius $R_{2} / A=r_{2}$ is fixed by the mass ratio of the components; approximately

$$
r_{2}=0.38-0.3 \log \left(m_{1} / m_{2}\right) \text {. }
$$

For AX Mon, we have probably a mass ratio of about 3 , so that $r_{2}=0.28$. If Be stars were built on a model similar to AX Mon, 28 out of each $100 \mathrm{Be}$ stars would display visible eclipses. Not much photometric work has been done systematically on $\mathrm{Be}$ stars, in particular on the faint ones, thus we have no good statistical surveys: nevertheless, the above figure is unacceptable and would simply mean that the binary model represents only a small fraction of all Be stars.

More plausible are models with mass ratios $m_{1} / m_{2}=10$ or even more. The fraction of eclipsing systems will then drop below $20 \%$. Moreover, the size of the emitting disk will then be conveniently large, for this dimension is probably given roughly by the radius of the critical Roche lobe about the accreting star, which is

$$
R_{\text {disk }}=A\left(0.38+0.2 \log \left(m_{1} / m_{2}\right)\right) \text {. }
$$

Nevertheless, I must admit that the simple calculation of probability of eclipses appears to me as a rather serious objection to the idea that most Be stars can be explained as interacting binaries. However, we have not really tried yet to develop and evolve binary models specifically meant to represent Be stars.

I think it is fair to conclude that at the present time, it is impossible to decide what fraction of $\mathrm{Be}$ stars are interacting binaries. Both competing models should be further studied, developed and tested.

\section{Acknowledgements}

My thanks are due to Ms Marietta L. Eaker for typing the manuscript. The work reported in this session of the Symposium by R. S. Polidan, G. J. Peters, and myself, has been to a large degree supported by NSF grant MPS 74-04194A01 (Popper/Plavec).

\section{References}

Bath, G. T.: 1969, Astrophys. J. 158, 571.

Bath, G. T.: 1972, Astrophys. J. 173, 121.

Batten, A. H.: 1974, Publ. Dom. Astrophys. Obs. 14, 191.

Batten, A. H., Fisher, W. A., Baldwin, B. W., and Scarfe, C. D.: 1975, Nature 253, 174.

Benson, R. S.: 1970, Bull. Am. Astron. Soc. 2, 295.

Biermann, P. and Hall, D. S.: 1973, Astron. Astrophys. 27, 249.

Ǩrî̌, S.: 1969, in M. Hack (ed.), Mass Loss from Stars, D. Reidel, Publ. Co., Dordrecht-Holland, p. 257.

Limber, D. N. and Marlborough, M. J.: 1968, Astrophys. J. 152, 181.

Lubow, S. H. and Shu, F. H.: 1975, Astrophys. J. 198, 383.

Paczyński, B.: 1971, Ann. Rev. Astron. Astrophys. 9, 183.

Plavec, M.: 1970a, in A. Slettebak (ed.), Stellar Rotation, D. Reidel, Publ. Co., Dordrecht-Holland, p. 133.

Plavec, M.: 1970b, Publ. Astron. Soc. Pacific 82, 957.

Plavec, M. and Horn, J.: 1969, in M. Hack (ed.), Mass Loss from Stars, Reidel, Publ. Co., DordrechtHolland, p. 242.

Plavec, M., Polidan, R. S., and Peters, G. J.: 1973, Bull. Am. Astron. Soc. 5, 413.

Plavec, M. and Polidan, R. S.: 1975, Nature 253, 173.

van den Heuvel, E. P. J.: in A. Slettebak (ed.), Stellar Rotation, Reidel, Publ. Co., Dordrecht-Holland, p. 178. 


\section{DISCUSSION}

Conti: Your argument about $V / R$ variations being of necessity due to a binary seem pretty conclusive. It could be that $V / R$ variations can come from non-mass exchange systems in some cases (e.g. $\phi$ Per), but $I$ think it is also true that not all Be stars show $V / R$ variations. Perhaps the $V / R$ variation stars are the binaries and those without this variation are single. A decision on duplicity would then be relatively straightforward.

Plavec: It would be excellent if your idea were correct. At this moment, I do not think we are able to decide.

Young: If the secondary star is in fact supplying most of the angular momentum to the Be star that must come out of the orbital angular momentum and so presumably the orbital period will change. Have you calculated or estimated if the changes might be observable over, say, a 10-year period?

Plavec: Yes. Calculations have been made. The big problem is whether some part of the material is leaving the system. This is a great unknown. If you play with that, unfortunately, you can get almost any numbers you like. But at least in the case of $\beta$ Lyr and U Cep, period changes have been detected and they are in agreement with what one can expect.

Slettebak: You mentioned mechanisms for getting the material out into the shell in the case of single stars, and there may be some difficulties with the steliar wind model. There was a suggestion made at the Stellar Rotation Colloquium in 1969 by Roxburgh (Stellar Rotation, ed. A. Slettebak, D. Reidel Publ. Co., Dordrecht-Holland, 1970, p. 19) in which he pointed out that the temperature and pressure in the equatorial regions of a rapidly rotating star will at some time resemble that in the atmospheres of pulsating stars. You will get an A-type giant atmosphere, as you have in RR Lyrae stars, and so a pulsational instability may set in which may eject material. I do not believe that this suggestion has ever been discussed in a quantitative way.

Plavec: I agree that this is one possibility. Incidentally I am not against the stellar wind explanation; I only think that it is incomplete. If there is a strong stellar wind blowing all the time, how do you explain that in spite of that there is an envelope around the star with a great outflow which stops and then falls back, in the case of $48 \mathrm{Lib}$, or as in the case of Pleione?

Slettebak: I think a second problem with the stellar wind is one that I referred to before. If you believe that gravity darkening takes place, then you have trouble explaining how the dark equatorial regions, as you would expect in the later Be stars, can produce a strong stellar wind. So I was just proposing this pulsation idea in order to get it out on the floor for discussion.

Cowley: If we accept Plavec's idea of the binaries being of two types, that is, one where there is still mass transfer going on and another type where the stars have evolved beyond that so that they do have helium cores and have gone to the stage where they can no longer transfer material, we can propose an observational test. For example, for the ones in which you now have a contracted core you no longer have a contact system and therefore you would expect that the emitting shell would always be constant. Therefore we should look in systems in which there are no known variations, where the helium and hydrogen emission does not come and go, for evidence of this kind of a companion; whereas in the ones where it comes and goes we should expect then only to find late-type subgiant companions.

Plavec: I agree completely. If there exist binary systems in which the mass transfer has terminated, the only change we should observe in the emitting disk would be its secular fading. 